\title{
Outbreaks of Foodborne Disease Associated with Fruit and Vegetable Juices, 1922-2019'
}

\author{
Matthew D. Krug, Travis K. Chapin, Michelle D. Danyluk, Renée M. Goodrich-Schneider, Keith \\ R. Schneider, Linda J. Harris, and Randy W. Worobo²
}

In response to several outbreaks of illness in the 1990s associated with raw juices processed at commercial facilities, the US Food and Drug Administration (FDA) introduced regulation (21 Code of Federal Regulations 120; FDA 2001) mandating that all $100 \%$ fruit/vegetable juices be produced under a Hazard Analysis and Critical Control Point (HACCP) plan. The juice HACCP regulation applies to domestic and imported $100 \%$ juice products and has implications for juice producers in countries that export juice to the United States.

HACCP plans must have supporting good manufacturing practices (GMPs) and sanitation standard operating procedures (SSOPs). In addition, the regulation requires juice processors apply a treatment that results in at least a 5 -log reduction of the "pertinent microorganism," which is defined as "the most resistant microorganism of public health significance that is likely to occur in the juice." Identification of the pertinent microorganism for a particular juice may be based upon foodborne illness outbreak data or other appropriate information such as survey or recall reports involving isolation of pathogens from juices or the fruits or vegetables used to produce those juices. Currently, Salmonella is generally accepted as the pertinent pathogen in citrus juices, whereas Escherichia coli O157:H7 as well as
Cryptosporidium parvum are both considered pertinent for apple juice (FDA 2001).

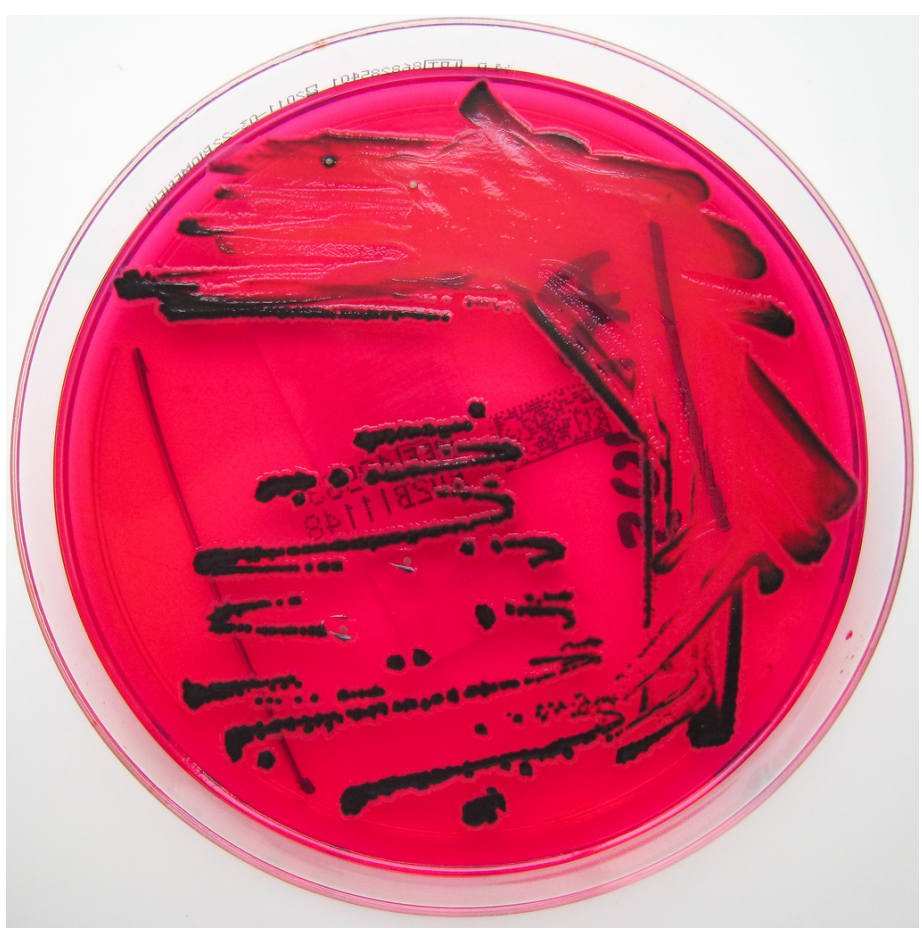

Figure 1. Salmonella species on X.L.D. agar.

Credits: Nathan Reading, CC BY-NC-ND 2.0 (http://flic.kr/p/9TtH1V)

1. This document is FSHN12-04, one of a series of the Food Science and Human Nutrition Department, UF/IFAS Extension. Original publication date January 2012. Revised June 2020. Visit the EDIS website at https://edis.ifas.ufl.edu for the currently supported version of this publication.

2. Matthew D. Krug, state specialized agent, food science, UF/IFAS Southwest Florida Research and Education Center; Travis K. Chapin, state specialized agent, food safety; Michelle D. Danyluk, professor, Food Science and Human Nutrition Department, UF/IFAS Citrus REC; Renée M. Goodrich-Schneider, professor; Keith R. Schneider, professor, Food Science and Human Nutrition Department; Linda J. Harris, cooperative Extension specialist-microbial food safety, Department of Food Science and Technology, University of California; and Randy W. Worobo, associate professor, Department of Food Science and Technology, Cornell University; UF/IFAS Extension, Gainesville, FL 32611.

The Institute of Food and Agricultural Sciences (IFAS) is an Equal Opportunity Institution authorized to provide research, educational information and other services only to individuals and institutions that function with non-discrimination with respect to race, creed, color, religion, age, disability, sex, sexual orientation, marital status, national origin, political opinions or affiliations. For more information on obtaining other UF/IFAS Extension publications, contact your county's UF/IFAS Extension office. U.S. Department of Agriculture, UF/IFAS Extension Service, University of Florida, IFAS, Florida A \& M University Cooperative Extension Program, and Boards of County Commissioners Cooperating. Nick T. Place, dean for UF/IFAS Extension. 
Outbreaks reported to the US Centers for Disease Control and Prevention (CDC) are compiled in the CDC National Outbreak Reporting System (NORS) database available at https://wwwn.cdc.gov/norsdashboard (CDC 2018). This tool was used, in part, for the preparation of the table presented here and may also be a useful resource when conducting a hazard analysis. A microbial risk assessment for unpasteurized fruit juices and cider prepared by Health Canada may also be a useful resource (Mihajlovic et al. 2013). This document is intended to highlight juice-related outbreaks, aid juice processors in the identification of "pertinent microorganisms," and review the locations, venues of juice preparations, and severity of juice-associated outbreaks.

\section{References}

Besser, R. E., S. M. Lett, J. T. Weber, M. P. Doyle, T. J. Barrett, J. G. Wells, and M. P. Griffin. 1993. "An outbreak of diarrhea and hemolytic uremic syndrome from Escherichia coli O157:H7 in fresh-pressed apple cider." Journal of the American Medical Association 269:2217-2220.

Birkhead, G. S., D. L. Morse, W. C. Levine, J. K. Fudala, S. F. Kondracki, H. G. Chang, M. Shaydgani, L. Novick, and P. A. Blake. 1993. "Typhoid fever at a resort hotel in New York: A large outbreak with an unusual vehicle." Journal of Infectious Diseases 167:1228-1232.

Blackburn, B. G., J. M. Mazurek, M. Hlavsa, J. Park, M. Tillapaw, M. Parrish, E. Salehi, W. Franks, E. Koch, F. Smith, L. Xiao, M. Arrowood, V. Hill, A. da Silva, S. Johnston, and J. L. Jones. 2006. "Cryptosporidiosis associated with ozonated apple cider.” Emerging Infectious Diseases 12:684-686.

Butler, M. E. 2000. "Salmonella outbreak leads to juice recall in Western states." Food Chemical News 42:19-20.

Buzby, J. C., and S. R. Crutchfield. 1999. "New Juice Regulations Underway." Food Review. http://ageconsearch.umn. edu/record/266215/files/FoodReview-218.pdf. Accessed August 7, 2018.

CDC (Centers for Disease Control and Prevention). 1975. "Salmonella typhimurium outbreak traced to a commercial apple cider-New Jersey." Morbidity and Mortality Weekly Report 24:87-88.

CDC. 1991. "Cholera associated with imported frozen coconut milk-Maryland." Morbidity and Mortality Weekly Report 40:843-844.
CDC. 1995. "Outbreak of Salmonella Hartford infections among travelers to Orlando, Florida." EPI-AID Trip Report 95-62.

CDC. 1996. "Outbreak of Escherichia coli O157:H7 infections associated with drinking unpasteurized commercial apple juice-British Columbia, California, Colorado, and Washington, October 1996." Morbidity and Mortality Weekly Report 45:975.

CDC. 1997. "Outbreaks of Escherichia coli O157:H7 infection and cryptosporidiosis associated with drinking unpasteurized apple cider-Connecticut and New York, October 1996." Morbidity and Mortality Weekly Report 46:4-8.

CDC. 1999. "Outbreak of Salmonella serotype Muenchen infections associated with unpasteurized orange juiceUnited States and Canada, June 1999." Morbidity and Mortality Weekly Report 48:582-585.

CDC. 2006. "Botulism associated with commercial carrot juice-Georgia and Florida." Morbidity and Mortality Weekly Report 55:1098-1099.

CDC. 2010. "Investigation Update: Multistate outbreak of human Typhoid Fever infections associated with frozen mamey pulp." http://www.cdc.gov/salmonella/typhoidfever/ index.html. Accessed August 7, 2018.

CDC. 2018. "National Outbreak Reporting System (NORS)." https://www.cdc.gov/nors/index.html. Accessed October 25, 2019.

CFIA (Canadian Food Inspection Agency). 2014. "Food Recall Warning - Unpasteurized apple cider processed by Rolling Acres Cider Mill recalled due to E. coli O157:H7." https://web.archive.org/web/20181204022225/http://www. inspection.gc.ca/about-the-cfia/newsroom/food-recallwarnings/complete-listing/2014-10-30/eng/1414720185030 /1414720197088. Accessed August 8, 2018.

Cody, S. H., K. Glynn, J. A. Farrar, K. L. Cairns, P. M. Griffin, J. Kobayashi, M. Fyfe, R. Hoffman, A. S. King, J. H. Lewis, B. Swaminathan, R. G. Bryant, and D. J. Vugia. 1999. "An outbreak of Escherichia coli O157:H7 infection from unpasteurized commercial apple juice." Annals of Internal Medicine 130:202-209.

Cook, K. A., T. E. Dobbs, W. G. Hlady, J. G. Wells, T. J. Barrett, N. D. Puhr, G. A. Lancette, D. W. Bodager, W. L. Toth, C. A. Genese, A. K. Highsmith, K. E. Pilot, L. Finelli, and D. L. Swerdlow. 1998. "Outbreak of Salmonella 
serotype Hartford infections associated with unpasteurized orange juice." Journal of the American Medical Association 280:1504-1509.

de Noya, B., Z. Díaz-Bello, C. Colmenares, R. RuizGuevara, L. Mauriello, R. Zavala-Jaspe, J. A. Suarez, T. Abate, L. Naranjo, M. Paiva, L. Rivas, J. Castro, J. Márques, I. Mendoza, H. Acquatella, J. Torres, and O. Noya. 2010. "Large urban outbreak of orally acquired acute Chagas disease at a school in Caracas, Venezuela." Journal of Infectious Disease 201:1308-1315.

Duncan, T. G., J. A. Coull, E. R. Miller, and H. Bancroft. 1946. "Outbreak of typhoid fever with orange juice as the vehicle illustrating the value of immunization." American Journal of Public Health 36:34-36.

Eisenstein, A. B., R. D. Aach, W. Jacobson, and A. Goldman. 1963. "An epidemic of infectious hepatitis in a general hospital." Journal of the American Medical Association 185:171-174.

FDA (US Food and Drug Administration). Federal Register Proposed Rules - 63 FR 20449 April 24, 1998 - HACCP; Procedures for the Safe and Sanitary Processing and Importing of Juice; Food Labeling: Warning Notice Statements; Labeling of Juice Products. Federal Register: April 24, 1998 63:20449-20486. https://www.federalregister.gov/ documents/1998/04/24/98-11025/hazard-analysis-andcritical-control-point-haccp-procedures-for-the-safe-andsanitary-processing-and. Accessed August 7, 2018.

FDA. Federal Register Final Rule - 66 FR 6137, January 19, 2001: Hazard Analysis and Critical Control Point (HACCP); Procedures for the Safe and Sanitary Processing and Importing of Juices. Federal Register: January 19, 2001 66:6137-6202.

Frank, C., J. Walter, M. Muehlen, A. Jansen, U. van Treeck, A. M. Hauri, I. Zoellner, E. Schreier, O. Hamouda, and K. Stark. 2005. "Large outbreak of hepatitis A in tourists staying at a hotel in Hurghada, Egypt, 2004 - orange juice implicated." Eurosurveillance 10:2720.

INS DOH (Indiana State Department of Health). Summary of Special Disease Outbreak Investigations-1997, Appendix E. https://web.archive.org/web/20170118103322/http:// www.in.gov/isdh/21186.htm. Accessed October 5, 2011.

IDPH (Iowa Department of Public Health). 2014. Iowa surveillance of notifiable and other diseases: Annual report
2013. http://publications.iowa.gov/17797/1/IDPH_Annual_Rpt_2013.pdf. Accessed August 7, 2018.

Jain, S., S. A. Bidol, J. L. Austin, E. Berl, F. Elson, M. L. Williams, M. Deassy III, M. E. Moll, V. Rea, J. D. Vojdani, P. A. Yu, R. M. Hoekstra, C. R. Braden, and M. F. Lynch. 2009. "Multistate outbreak of Salmonella Typhimurium and Saintpaul infections associated with unpasteurized orange juice-United States, 2005." Clinical Infectious Diseases 48:1065-1071.

Katz, D. J., M. A. Cruz, M. J. Trepka, J. A. Sarez, P. D. Filrella, and R. M. Hammond. 2002. "An outbreak of typhoid fever in Florida associated with an imported fruit." Journal of Infectious Diseases 186:234-239.

Krause, G., R. Terzagian, and R. Hammond. 2001. "Outbreak of Salmonella serotype Anatum infection associated with unpasteurized orange juice." Southern Medical Journal 94:1168-1172.

Lester, R., T. Stewart, J. Carnie, S. Ng, and R. Taylor. 1991. "Air travel-associated gastroenteritis outbreak, August 1991." Communicable Disease Intelligence 15:292-293.

LSDEPC (Laboratory Surveillance Data for Enteric Pathogens in Canada). "Annual summary 2005." https://web. archive.org/web/20141201115634/http://publications.gc.ca/ collections/collection_2007/phac-aspc/HP57-1-2005E.pdf. Accessed August 7, 2018.

Mihajlovic, B., B. Dixon, H. Couture, and J. Farber. 2013. "Qualitative microbiological risk assessment of unpasteurized fruit juice and cider." Int. Food Risk Anal. J. 3:5. https:// doi.org/10.5772/57161

Millard, P. S., K. F. Gensheimer, D. G. Addiss, D. M. Sosin, G. A. Beckett, A. Houck-Jankoski, and A. Hudson. 1994. "An outbreak of cryptosporidiosis from fresh-pressed apple cider." Journal of the American Medical Association 272:1592-1596.

MDH (Minnesota Department of Health). 2014. "Escherichia coli $\mathrm{O} 157$ infection and hemolytic uremic syndrome (HUS), 2011.” https://www.health.state.mn.us/diseases/ reportable/dcn/sum11/ecoli.html. Accessed August 7, 2018.

National Centre for Disease Control/Communicable Diseases Network Australia New Zealand, Australian Department of Health and Aged Care. 1999. "Salmonellosis outbreak, South Australia." Communicable Diseases Intelligence 23 (3): 73. 
Noël, H., A. Hofhuis, R. De Jonge, A. E. Heuvelink, A. De Jong, M. E. Heck, C. De Jager, and W. van Pelt. 2010. "Consumption of fresh fruit juice: How a healthy food practice caused a national outbreak of Salmonella Panama gastroenteritis." Foodborne Pathogens and Disease 7:375-381.

Paquet, P. 1923. "Épidémie de fièvre typhoïde: Déterminée par la consommation de petit citre." Revue d'Hygine 45:165-169.

Parish, M. E. 1998. "Coliforms, Escherichia coli, and Salmonella serovars associated with a citrus-processing facility implicated in a salmonellosis outbreak." Journal of Food Protection 61:280-284.

Parish, M. 2000. "Relevancy of Salmonella and pathogenic E. coli to fruit juices. Proceedings IFU-Workshop 'Microbiology." Fruit Processing 10:246-250.

Pereira, K. S., F. L. Schmidt, A. M. A. Guaraldo, R. M. B. Franco, V. L. Dias, and L. A. C. Passos. 2009. "Chagas' disease as a foodborne illness." Journal of Food Protection 72:441-446.

Schaffzin, J. K., F. Coronado, N. B. Dumas, T. P. Root, T. A. Halse, D. J. Schoonmaker-Bopp, M. M. Lurie, D. Nicholas, B. Gerzonich, G. S. Johnson, B. J. Wallace, and K. A. Musser. 2012. "Public health approach to detection of non-O157 Shiga toxin-producing Escherichia coli: summary of two outbreaks and laboratory procedures." Emerging Infectious Diseases 140 (2): 283-289. https://doi. org/10.1017/s0950268811000719

Singh, B. R., S. B. Kulshreshtha, and K. N. Kapoor. 1995. "An orange juiceborne outbreak due to enterotoxigenic Escherichia coli." Journal of Food Science and TechnologyIndia 34:504-506.

Steele, B. T., N. Murphy, and C. P. Rance. 1982. "An outbreak of hemolytic uremic syndrome associated with ingestion of fresh apple juice." Journal of Pediatrics 101:963-966.

Tabershaw, I. R., L. L. Schmelzer, and H. B. Bruhn. 1967. "Gastroenteritis from an orange juice preparation." Archives of Environmental Health 15:72-77.

Tamblyn, S., J. de Grosbois, D. Taylor, and J. Stratton. 1999. "An outbreak of Escherichia coli O157:H7 infection associated with unpasteurized non-commercial, custom-pressed apple cider - Ontario, 1998." Canada Communicable Disease Report 25:113-117; discussion 117-120.

Taylor, J. L., J. Tuttle, T. Pramukul, K. O’Brien, T. J. Barrett, B. Jolbaito, Y. L. Lim, D. J. Vugia, J. G. Morris, Jr., R. V. Tauxe, and D. M. Dwyer. 1993. "An outbreak of cholera in Maryland associated with imported commercial frozen fresh coconut milk." Journal of Infectious Diseases 167:1330-1335.

Thurston, H., J. Stuart, B. McDonnell, S. Nicholas, and T. Cheasty. 1998. "Fresh orange juice implicated in an outbreak of Shigella flexneri among visitors to a South African game reserve." Journal of Infectious Diseases 36:350.

Tsai, H.-C., S. Lee, C.-K. Huang, C.-M. Yen, E.-R. Chen, and Y.-C. Liu. 2004. "Outbreak of eosinophilic meningitis associated with drinking raw vegetable juice in southern Taiwan." American Journal of Tropical Medicine and Hygiene 71:222-226.

Vojdani, J., L. Beuchat, and R. Tauxe. 2008. "Juice-associated outbreaks of human illness in the United States, 1995 through 2005." Journal of Food Protection 71 (2): 356-364. 
Table 1. Outbreaks of human foodborne disease from various microorganisms associated with juices during the period of 1922-2019.

\begin{tabular}{|c|c|c|c|c|c|c|c|}
\hline Type & Product & Year & Pathogen $^{\mathrm{a}}$ & Location & Venue & Cases (Deaths) ${ }^{b}$ & Reference $^{c}$ \\
\hline \multirow[t]{2}{*}{ Açaí } & Unspecified & 2004 & Trypanosoma cruzid $^{\mathrm{d}}$ & Brazil & Retail & 27 & Pereira et al. 2009 \\
\hline & Unspecified & 2007 & Trypanosoma cruzi & Brazil & Retail & 25 & Pereira et al. 2009 \\
\hline \multirow[t]{24}{*}{ Apple } & Unpasteurized & 1922 & S. Typhi & France & $N R^{e}$ & $23(0)$ & Paquet 1923 \\
\hline & Unpasteurized & 1974 & S. Typhimurium & US (NJ) & $\begin{array}{l}\text { Farm, small } \\
\text { retail outlets }\end{array}$ & $296(0)$ & CDC 1975 \\
\hline & Unpasteurized & 1980 & E. coli O157:H7 (suspected) & Canada (ON) & Local market & $14(1)$ & Steele et al., 1982 \\
\hline & Unpasteurized & 1991 & E. coli O157:H7 & US (MA) & $\begin{array}{l}\text { Small cider } \\
\text { mill }\end{array}$ & $23(0)$ & Besser et al. 1993 \\
\hline & Unpasteurized & 1993 & Cryptosporidium & US (ME) & School & $213(0)$ & Millard et al. 1994 \\
\hline & Unpasteurized & 1996 & C.parvum & US (NY) & $\begin{array}{l}\text { Small cider } \\
\text { mill }\end{array}$ & $31(0)$ & CDC 1997 \\
\hline & Unpasteurized & 1996 & E. coli 0157:H7 & US (CT) & $\begin{array}{l}\text { Small cider } \\
\text { mill }\end{array}$ & $14(0)$ & CDC 1997 \\
\hline & Unpasteurized & 1996 & E.coli O157:H7 & US (WA) & $\begin{array}{l}\text { Small cider } \\
\text { mill }\end{array}$ & $6(0)$ & FDA 2001 \\
\hline & Unpasteurized & 1996 & E. coli O157:H7 & $\begin{array}{l}\text { Canada (BC), US } \\
(\mathrm{CA}, \mathrm{CO}, \mathrm{WA})\end{array}$ & Retail & $70(1)$ & CDC 1996, Cody et al. 1999 \\
\hline & Unpasteurized & 1997 & E. coli O157:H7 & US (IN) & Farm & 6 & INS DOH 1997 \\
\hline & Unpasteurized & 1998 & E. coli O157:H7 & Canada (ON) & Farm/Home & $14(0)$ & Tamblyn et al. 1999 \\
\hline & Unpasteurized & 1999 & E. coli $0157: \mathrm{H7}$ & US (OK) & NR & $25(0)$ & CDC 2018 \\
\hline & $\begin{array}{l}\text { Unpasteurized } \\
\text { (ozonated) }\end{array}$ & 2003 & C. parvum & US (OH) & Farm/Retail & $144(0)$ & $\begin{array}{l}\text { Blackburn 2006, Vojdani et al. 2008, CDC } \\
2018\end{array}$ \\
\hline & Unpasteurized & 2004 & E. coli 0111 and C. parvum & US (NY) & Farm/Home & $212(0)$ & $\begin{array}{l}\text { Vojdani et al. 2008, Schaffzin et al. 2012, } \\
\text { CDC } 2018\end{array}$ \\
\hline & Unpasteurized & 2005 & E. coli O157:H7 & Canada (ON) & NR & 4 & LSDEPC 2005 \\
\hline & Unpasteurized & 2007 & E. coli O157:H7 & US (MA) & NR & $9(0)$ & CDC 2018 \\
\hline & Unpasteurized & 2008 & E. coli 0157:H7 & US (IA) & Fair, festival & $5(0)$ & CDC 2018 \\
\hline & Unpasteurized & 2010 & E. coli O157:H7 & US (MD) & Retail & $7(0)$ & CDC 2018 \\
\hline & Unpasteurized & 2011 & $\begin{array}{l}\text { Cryptosporidium, E. coli } \\
\text { O111:NM }\end{array}$ & US (MN) & Farm & $14(0)$ & MDH 2014, CDC 2018 \\
\hline & Unpasteurized & 2011 & Cryptosporidium & US (OH) & NR & $4(0)$ & CDC 2018 \\
\hline & Unpasteurized & 2012 & E. coli O157:H7 & US (MI) & Home & $3(0)$ & CDC 2018 \\
\hline & Unpasteurized & 2013 & Cryptosporidium & US (IA) & Home & $10(0)$ & IDPH 2014, CDC 2018 \\
\hline & Unpasteurized & 2013 & Cryptosporidium & US (OH) & NR & $8(0)$ & CDC 2018 \\
\hline & Unpasteurized & 2013 & S. Typhimurium & US (PA) & NR & $10(0)$ & CDC 2018 \\
\hline
\end{tabular}




\begin{tabular}{|c|c|c|c|c|c|c|c|}
\hline Type & Product & Year & Pathogen $^{\mathrm{a}}$ & Location & Venue & Cases (Deaths) ${ }^{\mathrm{b}}$ & Reference $^{c}$ \\
\hline & Unpasteurized & 2014 & E. coli O157:H7 & Canada (ON) & $\begin{array}{l}\text { Farm, cider } \\
\text { mill, local } \\
\text { farmer's } \\
\text { market }\end{array}$ & 3 & CFIA 2014 \\
\hline & Unpasteurized & 2014 & Campylobacter jejuni & US (AZ) & Fair, festival & $6(0)$ & CDC 2018 \\
\hline & Unpasteurized & 2015 & E. coliO157 & US (MN) & Farm & $2(0)$ & CDC 2018 \\
\hline & Unpasteurized & 2015 & E. coli $\mathrm{O} 45$ & US (MI) & NR & $2(0)$ & CDC 2018 \\
\hline & Unpasteurized & 2016 & E. coli O157:H7 & US (KS) & Fair, festival & $56(0)$ & CDC 2018 \\
\hline & Unspecified & 2015 & E. coliO111:NM & US (CA) & NR & $15(0)$ & CDC 2018 \\
\hline \multirow[t]{2}{*}{ Carrot } & Homemade & 1993 & C. botulinum & US (WA) & Home & $1(0)$ & Buzby and Crutchfield 1999 \\
\hline & Pasteurized & 2006 & C. botulinum & US (FL, GA) & Retail & $4(1)$ & CDC 2006, CDC 2018 \\
\hline Coconut & Milk ${ }^{f}$ & 1991 & Vibrio cholerae & US (MD) & Home/picnic & 4 & CDC 1991, Taylor et al. 1993 \\
\hline Guava & Unspecified & 2007 & Trypanosoma cruzi & Venezuela & School & $103(1)$ & de Noya et al. 2010 \\
\hline \multirow[t]{2}{*}{ Mamey } & Frozen Puree & 1999 & S. Typhi & US (FL) & NR & $16(0)$ & Katz et al. 2002, CDC 2018 \\
\hline & Frozen Pulp & 2010 & S. Typhi & US (CA, NV) & Restaurant & $12(0)$ & CDC 2010, CDC 2017 \\
\hline \multirow[t]{3}{*}{ Mixed Fruit } & Unspecified & 2002 & Shigella sonnei & $\begin{array}{l}\text { Canada, US, } \\
\text { UK, British West } \\
\text { Indies }\end{array}$ & Resort & 78 & CDC 2018 \\
\hline & Açaí, sugar cane & 2006 & Trypanosoma cruzi & Brazil & NR & $94(6)$ & Pereira et al. 2009 \\
\hline & $\begin{array}{l}\text { Açaí, banana, } \\
\text { strawberry, sugar } \\
\text { cane }\end{array}$ & 2007 & Hepatitis A & US (FL) & Restaurant & $3(0)$ & CDC 2018 \\
\hline $\begin{array}{l}\text { Mixed Fruit / } \\
\text { Vegetable }\end{array}$ & $\begin{array}{l}\text { Clover, sweet potato } \\
\text { leaf, apple }\end{array}$ & 2001 & $\begin{array}{l}\text { Angiostrongylus } \\
\text { cantonesis }^{9}\end{array}$ & Taiwan & Home & 5 & Tsai et al. 2004 \\
\hline \multirow[t]{2}{*}{ Orange } & Unpasteurized & 1992 & Enterotoxigenic E. coli & India & $\begin{array}{l}\text { Roadside } \\
\text { Vendor }\end{array}$ & $6(0)$ & Singh et al. 1995 \\
\hline & Unpasteurized & 1995 & $\begin{array}{l}\text { Salmonella Gaminera, } \\
\text { Hartford and Rubislaw }\end{array}$ & US (FL) & Retail & $63(0)$ & CDC 1995, Cook et al. 1998, Parish 1998 \\
\hline & Unpasteurized & 1995 & Shigella flexneri & South Africa & Restaurant & 14 & Thurston et al. 1998 \\
\hline & Unpasteurized & 1999 & S. Anatum & US (FL) & $\begin{array}{l}\text { Roadside } \\
\text { stand }\end{array}$ & $10(0)$ & Krause et al. 2001, CDC 2018 \\
\hline & Unpasteurized & 1999 & S. Typhimurium & Australia & Retail & $405(0)$ & National Centre for Disease Control 1999 \\
\hline & Unpasteurized & 2000 & S. Enteritidis & US (6 states) & $\begin{array}{l}\text { Retail and } \\
\text { Food Service }\end{array}$ & 88 & Butler 2000, CDC 2018 \\
\hline & Unpasteurized & 2004 & Hepatitis A & Egypt & Hotel & 351 & Frank et al. 2005 \\
\hline & Unpasteurized & 2005 & $\begin{array}{l}\text { S.Typhimurium and } S \text {. } \\
\text { Saintpaul }\end{array}$ & US (23 states) & $\begin{array}{l}\text { Retail and } \\
\text { Food Service }\end{array}$ & $157(0)$ & Jain et al. 2009, CDC 2018 \\
\hline
\end{tabular}




\begin{tabular}{|c|c|c|c|c|c|c|c|}
\hline Type & Product & Year & Pathogen ${ }^{a}$ & Location & Venue & Cases (Deaths) $^{\mathrm{b}}$ & Reference $^{c}$ \\
\hline & Unpasteurized & 2008 & S. Panama & $\begin{array}{l}\text { The } \\
\text { Netherlands }\end{array}$ & Retail & 33 & Noël et al. 2010 \\
\hline & Reconstituted & 1944 & S. Typhi & US (OH) & Hotel & $18(1)$ & Duncan et al. 1946 \\
\hline & Reconstituted & 1962 & Hepatitis A & US (MO) & Hospital & 24 & Eisenstein et al. 1963 \\
\hline & Reconstituted & 1965 & Unknown & US (CA) & $\begin{array}{l}\text { Football } \\
\text { game }\end{array}$ & 563 & Tabershaw et al. 1967 \\
\hline & Reconstituted & 1989 & S. Typhi & US (NY) & Hotel & 69 & Birkhead et al. 1993 \\
\hline & Unspecified & 1991 & Norwalk-like virus & Australia & Airline & 3,053 & Lester et al. 1991 \\
\hline Sugarcane & Unspecified & 2005 & Trypanosoma cruzi & Brazil & $\begin{array}{l}\text { Roadside } \\
\text { kiosk }\end{array}$ & $25(3)$ & Pereira et al. 2009 \\
\hline Watermelon & Homemade & 1993 & Salmonella spp. & US (FL) & Home & $18(0)$ & FDA 1998 \\
\hline \multicolumn{8}{|c|}{ 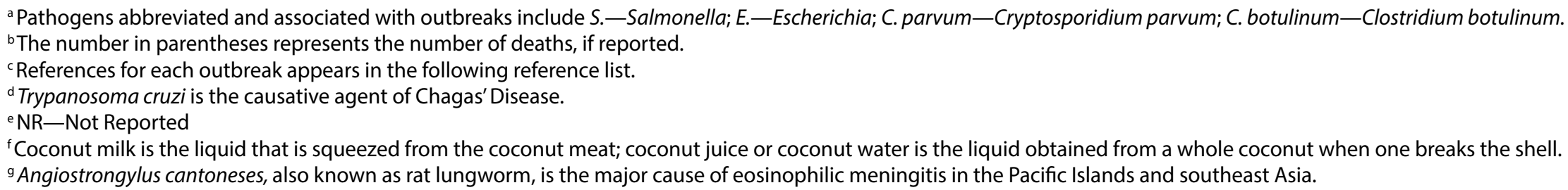 } \\
\hline
\end{tabular}

